\title{
Clinical importance of left atrial infiltration in cardiac transthyretin amyloidosis
}

Short Title: Left atrial infiltration in ATTR-CM

Francesco Bandera ${ }^{\mathrm{a}, \mathrm{b} *}$ MD PhD, Raffaele Martone ${ }^{\mathrm{c} *}$ MD, Liza Chacko ${ }^{\mathrm{d}}$ MD, Sharmananthan Ganesananthan ${ }^{\mathrm{d}}$ MS, Janet A Gilbertson ${ }^{\mathrm{d}}$ CSci, Markella Ponticos ${ }^{\mathrm{d}}, \mathrm{PhD}$ Thirusha Lane ${ }^{\mathrm{d}} \mathrm{RN}$ PhD, Ana Martinez-Naharro ${ }^{\mathrm{d}}$ MD PhD, Carol Whelan ${ }^{\mathrm{d}} \mathrm{MD}$, Cristina Quarta ${ }^{\mathrm{d}}$ MD PhD, Dorota Rowczenio ${ }^{\mathrm{d}} \mathrm{PhD}$, Rishi Patel, ${ }^{\mathrm{d}} \mathrm{MD}$, Yousuf Razvi, ${ }^{\mathrm{d}}$ MD Helen Lachmann ${ }^{\mathrm{d}} \mathrm{MD}$, Ashutosh Wechelakar ${ }^{\mathrm{d}}$ MD, James Brown ${ }^{\mathrm{d}}$ MD, Daniel Knight ${ }^{\mathrm{d}}$ MD, James Moon ${ }^{\mathrm{e}}$ MD, Aviva Petrie ${ }^{\mathrm{f}} \mathrm{PhD}$, Francesco Cappelli ${ }^{\mathrm{c}}$ MD, Marco Guazzi ${ }^{\mathrm{a}, \mathrm{b}}$ MD PhD, Luciano Potena ${ }^{\mathrm{g}}$ MD, Claudio Rapezzi ${ }^{\mathrm{h}, \mathrm{i}}$ MD, Ornella Leone ${ }^{\mathrm{g}}$ MD, Philip N Hawkins ${ }^{\mathrm{d}}$ MD PhD, Julian D Gillmore ${ }^{\mathrm{d} \bullet}$ MD PhD, Marianna Fontana $^{\mathrm{d} \bullet} \mathrm{MD} \mathrm{PhD}$

*Francesco Bandera and Raffaele Martone contributed equally to this work.

-Julian Gillmore and Marianna Fontana contributed equally to this work.

${ }^{a}$ Cardiology University Department, Heart Failure Unit, IRCCS Policlinico San Donato, San Donato Milanese, Milan, Italy;

${ }^{b}$ Department for Biomedical Sciences for Health, University of Milano, Milan, Italy.

${ }^{\mathrm{c}}$ Tuscan Regional Amyloid Center, Careggi University Hospital (AOUC), Florence, Italy

${ }^{\mathrm{d} N a t i o n a l ~ A m y l o i d o s i s ~ C e n t r e, ~ U n i v e r s i t y ~ C o l l e g e ~ L o n d o n, ~ R o y a l ~ F r e e ~ C a m p u s, ~ L o n d o n, ~ U K ~}$

e'Barts Heart Centre, St Bartholomew's Hospital, West Smithfield, London, UK

${ }^{\mathrm{f}}$ Eastman Dental Institute, University College London, Grays Inn Road, London WC1X 8LD, UK ${ }^{\mathrm{g}}$ Academic Hospital S. Orsola-Malpighi, Bologna, Italy.

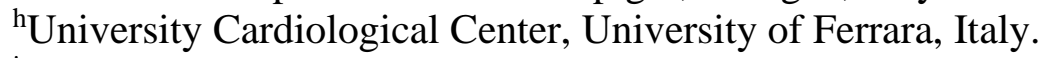

${ }^{\mathrm{i}}$ Maria Cecilia Hospital, GVM Care \& Research, Cotignola (RA), Italy.

Address for correspondence:

Dr Marianna Fontana,

National Amyloidosis Centre,

University College London, Royal Free Campus,

Rowland Hill Street, London, NW3 2PF, UK

E-mail: m.fontana@ucl.ac.uk

Phone No: +44 2074332764

Fax No: +44 2044332817

AKNOWLEDGMENTS: None.

SOURCE OF FUNDING: M Fontana is supported by a British Heart Foundation Intermediate Clinical Research Fellowship (FS/18/21/33447).

DISCLOSURES: None. 


\begin{abstract}
BACKGROUND: The clinical significance of left atrial (LA) involvement in ATTR amyloidosis cardiomyopathy (ATTR-CM) is of great clinical interest.

OBJECTIVES: The aims of this study were to characterize: (1)LA pathology in explanted ATTRCM hearts; (2)LA mechanics using echocardiographic speckle-tracking in a large cohort of ATTRCM patients; (3)to study the association with mortality.

METHODS: Congo red staining and immunohistochemistry was performed to assess the presence, type and extent of amyloid and associated changes in 5 explanted ATTR-CM atria. Echo speckletracking was used to assess LA reservoir, conduit, contractile function and stiffness in 906 ATTRCM patients (551 wt-ATTR-CM;93 T60A-ATTR-CM;241 V122I-ATTR-CM;21 other).

RESULTS: There was extensive ATTR amyloid infiltration in the 5 atria with loss of normal architecture, vessels remodelling, capillary disruption and subendocardial fibrosis. Echo speckletracking in 906 ATTR-CM patients demonstrated increased atrial stiffness [median(25th-75th quartile) 1.83(1.15-2.92)] that remained independently associated with prognosis, after adjusting for known predictors (lnLA stiff: HR 1.23; 95\%CI 1.03 -1.49 p=0.029). There was substantial impairment of the three phasic functional atrial components [reservoir 8.86(5.94-12.97)\%; conduit 6.5(4.53-9.28)\%; contraction function 4.0(2.29-6.56)\%]. Atrial contraction was absent in $22.1 \%$ of patients whose ECG showed sinus rhythm (SR)-“atrial electro-mechanical dissociation"(AEMD). AEMD was associated with poorer prognosis compared to SR patients with effective mechanical contraction ( $\mathrm{p}=0.0018$ ). AEMD conferred a similar prognosis to patients in atrial fibrillation.
\end{abstract}

CONCLUSIONS: The phenotype of ATTR-CM includes significant infiltration of the atrial walls with progressive loss of atrial function and increased stiffness, which is a strong independent predictor of mortality. AEMD emerged as a distinctive phenotype identifying patients in SR with poor prognosis.

KEYWORDS: Amyloidosis; Atrial function; Atrial stiffness; Echocardiography; Atrial strain; Atrial histology

\author{
ABBREVIATIONS: \\ ATTR-CM: Cardiac ATTR amyloidosis \\ wtATTR: wild-type transthyretin \\ hATTR: hereditary ATTR amyloidosis \\ HF: heart failure \\ LA: left atrium \\ LV: left ventricle \\ EMB: endomyocardial biopsy \\ ROI: region of interest \\ MCF: myocardial contraction factor \\ MAPSE: mitral annular plane systolic excursion \\ TAPSE: tricuspid annular plane systolic excursion \\ PASP: pulmonary artery systolic pressure
}




\section{INTRODUCTION}

ATTR-CM is a restrictive cardiomyopathy caused by extracellular deposition of amyloid fibrils derived from plasma transthyretin. It is most commonly non-hereditary, associated with the deposition of wtATTR, but there are numerous transthyretin gene variants associated with hATTR forms. Advances in imaging techniques(1)'(2)'(3)'(4) have led to validation of non-biopsy diagnostic criteria for ATTR-CM(5),(4), underscoring a recent exponential rise in diagnosis of ATTR-CM throughout the world.(6) ATTR-CM has emerged as a much under-diagnosed and under-recognized cause of HF.(7)

Studies in patients with cardiac ATTR amyloidosis have predominantly focused on the functional and structural consequences of amyloid infiltration within the ventricles, (8)'(9) causing biventricular wall thickening with non-dilated chambers, systolic and diastolic dysfunction and low cardiac output.(10)'(11) By contrast, assessment of the atria has focused mainly on atrial dimensions. LA dimension is generally considered an adequate measure of the cumulative effect of LV filling pressure over time, being a marker of severity and chronicity of diastolic dysfunction and resulting elevation of LA pressure.

However, measurement of LA dimension does not provide information on atrial function, which is emerging as a key component of overall cardiac performance in various cardiac disorders.(12) Echo speckle tracking now has an established role in the characterization of atrial function(13) and can assess the ability of the atria to expand during ventricular systole (reservoir function), the early-diastolic emptying (conduit function), the atrial shortening (atrial contraction) and, when associated with the estimation of left ventricle (LV) pressures, the resistance to deformation of the LA (LA stiffness).(14) Preliminary findings in cardiac amyloidosis have demonstrated impaired atrial systolic contraction,(15) but also loss of reservoir function, resulting in an atrium that acts merely as a conduit throughout the cardiac cycle.(16)'(17) To date, only small retrospective speckle tracking 
studies have been conducted in ATTR amyloidosis, and along with little in the way of histologic evaluation of the atrial wall, the significance of atrial infiltration, remodelling and dysfunction in the pathophysiology of cardiac ATTR amyloidosis is not clear.

The aims of the present study were to characterize (1) the spectrum of changes in LA structure in post mortem specimens of patients with ATTR-CM, (2) the functional consequences of amyloid deposition on LA stiffness and mechanics in a large cohort of patients with cardiac ATTR amyloidosis using echocardiographic speckle tracking, and (3) to assess the association between atrial functional parameters and clinical outcomes.

\section{METHODS}

\section{Patient Population}

Patients referred to the National Amyloidosis Centre (NAC), Royal Free Hospital, London, United Kingdom between 2000 and 2019 in whom ATTR-CM was confirmed on the basis of validated diagnostic criteria(5) were invited to participate in a prospective registry with protocolised clinical follow up program comprising systematic evaluation of cardiac parameters and survival. Clinical, biochemical and imaging data were retrospectively analysed for this study. Briefly, the diagnosis of ATTR-CM was established on the basis of the following criteria: presence of symptoms of heart failure together with an echocardiogram consistent with amyloidosis and either 1) direct EMB proof of ATTR amyloid, or 2) presence of ATTR amyloid in an extra-cardiac biopsy along with cardiac uptake on 99mTechnetium labelled 3,3-diphosphono-1,2-propanodicarboxylic acid(99mTc-DPD) scintigraphy, or 3) Perugini grade 2 or 3 cardiac uptake on 99mTc-DPD scintigraphy in the absence of a monoclonal immunoglobulin disorder.(5) The TTR gene was sequenced in all participants. Patients who had received disease modifying therapy including liver transplantation for hATTR amyloidosis, or a TTR-lowering therapy (within the context of a clinical trial) were excluded from the study. 
The atria from 5 explanted hearts of patients with ATTR-CM (3 with wtATTR-CM and 2 with hATTR-CM) were sampled.

Patients were managed in accordance with the Declaration of Helsinki and provided written informed consent (ref: 06/Q0501/42 and 79/2014/U/Sper, Royal Free Ethics Committee, London UK and AOU of Bologna, Italy).

\section{Histological analysis}

The atria samples were fixed in neutral buffered formalin and processed into a paraffin blocks (FFPE) for routine histology, Congo red staining and immunohistochemistry (IHC). IHC was performed using commercially available monospecific antibodies against human serum AA, kappa, lambda and pre-albumin (transthyretin), (Agilent), and NKx2.5 (Santa Cruz). Staining was performed on a manual platform, with Impress ${ }^{\mathrm{TM}}$ detection kits and a metal enhanced DAB substrate kit for visualizing the immuno compound. Ready to use antibodies against vWF, CD31, CD45 and CD68 (Leica microsystems) were carried out using a BOND Max immunostainer and DAB refine kit. Amyloid was laser captured for proteomic analysis to confirm IHC staining. Proteomic analysis was processed on the Thermo Scientific ${ }^{\mathrm{TM}} \mathrm{Q}-\mathrm{Exactive}$ Plus Orbitrap. Data was analysed using Mascot software and the Swiss-Prot human database. All IHC slides and proteomic analysis were interpreted blind to any clinical details by two independent reporters. IHC results were compared with data obtained from proteomic analysis in particular with respect to positive identification of the amyloid fibril protein.

\section{Echocardiography}

All echocardiograms were reviewed by experienced operators and analysed according to guidelines, as previously reported.(10) For the purpose of our analysis, we considered valvular regurgitation to be clinically significant if at least mild-to-moderate or greater.

\section{LA assessment}

LA dimensions were reported as parasternal long-axis diameter, absolute and BSA-indexed 4-chambers area. LA speckle tracking analysis was performed using GE EchoPAC software 
(Version 203), according with the current consensus document.(18) Briefly, non-foreshortened 2D apical 4-chamber view was used to define a $3 \mathrm{~mm}$-thickened wall region of interest (ROI) along the LA. The LA contour was extrapolated excluding pulmonary veins and LA appendage. Quality control check was performed to reject cases with significant ( $>1 / 3$ of LA contour) drop out of atrial wall. Zero-baseline was defined as ventricular end-diastole, using the R-R cycle for analysis. LA myocardial deformation was assessed as global longitudinal strain obtained with the endocardial curve of the ROI. Reservoir, contraction and conduit phase were studied respectively as: LA strain (LAS) reservoir= peak value at the onset of LV filling, LAS contraction= peak value at the onset of atrial contraction (for subjects on sinus rhythm) and LAS conduit= difference between LAS reservoir and LAS contraction (for subjects on sinus rhythm). LA stiffness (LA Stiffness) was calculated as the ratio between E/e' and LAS reservoir, where e' is the mean value between lateral and septal e'.(14)

\section{Statistical analysis}

All mortality data were obtained via the UK Office of National Statistics. The mortality endpoint was defined as time to death from baseline for all deceased patients and time to Censor date, $24^{\text {th }}$ of April 2019, from baseline among the remainder. Baseline was the time of diagnosis. The three genotypic sub-groups of interest were wtATTR-CM, V122I-associated hereditary ATTRCM (V122I-hATTR-CM) and T60A-associated hereditary ATTR-CM (T60A-hATTR-CM)

As a number of the numerical variables had skewed distributions, a Kruskal-Wallis test was used to compare the distributions of each of the numerical variables at baseline in the subgroups. A significant result was followed by Bonferroni corrected Mann-Whitney pairwise comparisons to establish where the differences lay. For categorical variables Chi-squared test was used followed by z-test with Bonferroni correction for pairwise comparison, due to the large sample size. Correlation between non-parametric variables was explored using Spearman test.

Survival was evaluated with Cox proportional hazards regression analysis, providing estimated hazard ratios with 95\% confidence intervals (CIs) and Kaplan-Meier curves. Twenty 
echocardiographic variables were selected based upon clinical relevance and previous report:(10) interventricular septum in diastole (IVSd), relative wall thickness (RWT), stroke volume indexed, LV ejection fraction (EF), LV longitudinal strain (LS), E/e', MCF, MAPSE, significant mitral regurgitation (MR), significant tricuspid regurgitation (TR), right atrium area (RAA) index, TAPSE, TAPSE/PASP, LA diameter, LA area, LA area indexed, LAS reservoir, LAS conduit, LAS contraction, and ln LA Stiffness. The proportional hazards assumption was checked and confirmed. The echocardiographic variables were first explored with univariate Cox regression analysis. The variables that were statistically significant predictors of outcome on simple Cox regression analysis were entered into a multivariable Cox proportional hazards analysis to determine which covariates were independent predictors of mortality. Because LA stiffness was introduced in the model, LAS reservoir and E/e' were not included for the collinearity between variables. The model was also adjusted for heart rate, cardiac rhythms, genotypes and time of diagnosis (the variable was categorized as before or after the 15 February 2015, which was the median date of diagnosis). Possible collinearity among candidate predictors was assessed using variance inflation factors with threshold equal to 5 .

After verifying the required statistical assumptions, a linear regression for LA reservoir function was assessed with diastolic variables, to understand the interplay between LA mechanics and LV diastolic function.

All data were analysed using Stata software (StataCorp.2017. Stata Statistical Software:Release 15. College Station, TX:StataCorp LLC). A significance level of 0.05 was used for all hypothesis tests unless otherwise stated.

\section{RESULTS}

\section{Characteristics of the overall cohort}

The echocardiograms of 1240 patients with ATTR-CM were retrospectively analysed by speckle tracking analysis of LA strain. Nine-hundred and six patients had LA strain analysis in line with current consensus guidelines.(18) In 334(26.9\%) patients, LA strain analysis was not feasible 
due to inadequate acoustic windows, excessive breathing-related cardiac motion, LA foreshortening or significant atrial wall drop out. The final cohort (Table 1 and 2) comprised 906 patients, 551(62.3\%) with wtATTR-CM [age 77(SD 7), male 95.3\%)], 241(26.6\%) with V122I-associated hATTR-CM [age 76(7), male 70.1\%], 93(10.3\%) with T60A-associated hATTR-CM [age 66(SD 7),male 65.5\%], 21(2.3\%) with non-V122I, non-T60A-associated hATTR-CM (age 62(SD 12), male $81 \%$ ). The patients with non-V122I, non-T60A-associated hATTR-CM had the following mutations: G47V,V30M,S77Y,I73V,A120S,D39V,E89K,H90D,I107V,F33I,V20I.

\section{Histological findings}

The 5 patients with ATTR-CM whose explanted hearts underwent histologic study were males, average age 61(range 51-88): 3 had wtATTR, 2 hATTR (V122I, E89Q). Figure 1 summarises the major histologic findings. There was extensive ATTR amyloid deposition within the myocardial interstitium and subendocardium in all samples. Proteomic analysis confirmed the amyloid deposits to be ATTR type and also identified ANP in 3 out of 5 patients, although with low Mascot score. Amyloid deposits were severe and diffusely distributed in 3 cases and moderate and scattered in multifocal areas in 2 cases. In all specimens, the myocardial interstitial deposits were peri-myocite and for the most part aggregated in nodules replacing normal tissue; in the subendocardium there were nodular deposits within fibrous tissue. Amyloid vascular deposits were also seen in all samples, both in small intramural vessels and in subepicardial arteries and veins, with a focal distribution in 4 cases and multifocal in 1 . In addition, phenotypic modulation and vascular remodelling in arteries of all samples were found as confirmed by high level of NKX2-5 expression in intimal and medial layers. NKX2-5 expression is not present in healthy adult vessels, as expression of NKX2-5 is associated with phenotypic modulation of vascular smooth muscle cells and endothelial cells of vessels undergoing vascular remodelling in pathology. Mild to moderate subendocardial fibrosis was present in all samples; myocardial interstitial fibrosis was absent.

Widespread ATTR amyloid deposition was associated with histologic myocardial remodelling in all samples, including myocyte morphologic changes such as attenuation/atrophy 
and thinning, which was distributed diffusely in 3 specimens and was multifocal in 2.

Focal/multifocal areas with reactive hypertrophied or vacuolated myocytes were also noted. Acute myocyte injury was absent in all samples.

CD31 and von Willebrand factor VIII immunostaining revealed decreased capillary network in all samples, with diffuse distribution in 3 specimens, multifocal in 1 and focal in 1 . There were no significant inflammatory infiltrates, with only two samples showing mild inflammation comprising interstitial macrophages and few lymphocytes.

\section{Strain derived LA structure and function.}

LA stiffness was significantly increased in patients with cardiac amyloidosis [LA stiffness (median $\left(25^{\text {th }}-75^{\text {th }}\right.$ quartile $\left.), 1.83(1.15-2.92)\right]$ compared to normal reference range [mean(SD), $0.21 \pm 0.1](19)$ and this was confirmed across the three predominant genotypes [WtATTR-CM (median(25 $5^{\text {th }}-75^{\text {th }}$ quartile) $1.72(1.10-2.83)$, T60A-ATTR-CM 1.67(0.87-2.70) and V122I-ATTRCM 2.12(1.34-3.29) Kruskal-Wallis,p=0.001, WtATTR-CM vs V122I-ATTR-CM,p=0.003, T60AATTR-CM vs V122I-ATTR-CM,p=0.024, other comparison not significant]. The amyloidosis phenotype was also characterized by significant reduction of the three atrial functional components [LAS reservoir (median $\left(25^{\text {th }}-75^{\text {th }}\right.$ quartile) $8.86(5.94-12.97)$, LAS conduit 6.5(4.53-9.28) and LAS contraction 4.0(2.29-6.56)], with significant differences across the three main genotypes. The most impaired overall functional pattern was present in patients with V122I genotype (Table 3). There was a weak correlation between the lnLA stiffness and atrial dilatation (LA indexed area) (Spearman $\left.\mathrm{r}^{2}=0.036, \mathrm{p}<0.001\right)$. Increased LA stiffness was associated with a progressive reduction in the reservoir, conduit and contraction function (Figure 2). Linear regression for LAS reservoir showed very low $\mathrm{r}$ for all LV diastolic variables (Supplemental Table 2). Among the 906 patients, 564(62.2\%) patients were in sinus rhythm (SR group) and 342 patients (37.8\%) were not (Non-SR group) comprising 313(91.5\%) in atrial fibrillation (AF) and 29(8.5\%) in atrial flutter or tachycardia. 
Of the patients in SR, 439(77.8\%) patients had evidence of atrial contraction on LA strain analysis (SR with LA mechanical contraction group-LAMC group) and 125(22.2\%) patients did not show evidence of atrial contraction (SR without LAMC group)(Figure 3). These groups were defined based only on the presence or absence of atrial contraction on LA strain analysis. Patients in the SR without LAMC group did not show evidence of A wave or the A wave was very low $(<20$ $\mathrm{cm} / \mathrm{s}$ ) on trans-mitral Doppler analysis. The clinical phenotypes (as identified by NAC staging system, NTproBNP, troponin, eGFR and 6MWT) of SR without LAMC and Non-SR patients were similar and significantly worse than patients in SR with LAMC (Table 1). SR without LAMC patients had more severe systolic dysfunction compared to patients in SR with LAMC. LA indexed area of SR with LAMC patients was significantly reduced only when compared to Non-SR group (Table 2). By contrast, LAS reservoir and LA stiffness were significantly and similarly impaired in SR without LAMC and Non-SR patients.

Fifty-seven percent of Non-SR patients were on anticoagulants, whilst both SR sub-groups showed a similar prevalence around $26-27 \%$.

\section{Strain derived LA structure, function and prognosis}

At follow up (mean 35(SD22) months) 370 (40.8\%) of 906 patients had died. Median patient survival from diagnosis by Kaplan-Meier analysis was 55 months in the overall group of SR patients, 58 and 44 in SR with LAMC and SR without LAMC, respectively, and 51 months in NonSR patients (Figure 4).

Twenty-one echocardiographic variables, heart rate, rhythm, genotype and the time of diagnosis were explored in the univariate Cox regression analysis (Supplemental Table 1). Ten echocardiographic variables and heart rate, cardiac rhythm, genotype and the time of diagnosis were entered into a multivariable Cox proportional hazard analysis (Table 4). In order to avoid collinearity and in light of a non-significant role in previous multivariate models,(10) E/e' was not entered into the multivariate model. For all variables the variance inflation factor was $<2$. The final model, combining IVSd, RWT, SVindex, LVLS, RAA index, significant MR, significant TR, 
TAPSE/PASP, LAA index, ln LA stiffness, heart rate, SR versus Non-SR and the time of diagnosis revealed that ln LA stiffness (HR 1.23; 95\%CI $1.03-1.49 \mathrm{p}=0.029$ ) remained independently associated with patient survival together with RAA index (HR:1.05;95\%,CI 1.01-1.10 p=0.033), LVLS (HR:1.07;95\%CI 1.03-1.12,p=0.002), significant MR (HR:1.35;95\%CI, 1.03- 1.77,p=0.032), genotypes (V122I-ATTR-CM vs WtATTR-CM: HR 1.49 95\% CI 1.12-1.97 p=0.006) and the period of diagnosis (Before vs after 09 February 2015 HR:1.52;95\%CI 1.13-2.06,p=0.006).

\section{DISCUSSION}

This is the first study to provide a systematic assessment of LA function and structure in patients with Cardiac ATTR amyloidosis. The disease is characterized on histology by extensive amyloid infiltration in the atria causing loss of normal architecture, remodelling of the vessels, capillary disruption and upregulation of the collagen at the level of the subendocardium. Atrial infiltration by ATTR amyloid translates in significantly increased atrial stiffness, which is independently associated with reduced outcome after adjusting for all known prognostic variables (Central Figure).

Abnormal stiffness of the LA myocardium is associated with reduction in the reservoir and contractile function of the atrium, with a remarkable fifth of patients showing an absence of contraction whilst remaining in sinus rhythm on the ECG, i.e. "atrial electro-mechanical dissociation" (AEMD). The prognosis of patients with AEMD was significantly poorer than for patients in sinus rhythm who maintained effective mechanical contraction, and was similar to those with AF.

Cardiac amyloidosis is considered an exemplar of restrictive cardiomyopathy, with ventricular diastolic dysfunction having the central role in disease pathophysiology and evolution. It has been thought that increasing LV infiltration by amyloid underlies progressive worsening of diastolic function, predominantly causing abnormal relaxation in the early stage leading to a shift of diastolic filling during late diastole.(20) As amyloid accumulates, LA pressure increases producing 
a pseudonormal pattern on the Doppler tracing. In more advanced disease, the increase in LV myocardial stiffness causes restriction to filling with a greater rise of ventricular pressure for a small change in volume, reflected in a characteristic elevation of the E/A ratio above 2. This pattern has been traditionally thought to reflect the restrictive ventricular physiology, i.e. rapid filling of a very stiff LV. However, an elevated E/A ratio, could also reflect a decrease of atrial contractile properties,(21) resulting from a primary LA systolic failure caused by the increased stiffness produced directly by atrial amyloid infiltration. Although it may be difficult to determine whether a diminutive transmitral A-wave is a function of the true atrial dysfunction or of a restrictive LV pathophysiology, it is likely that there is at least a component of primary atrial dysfunction. This hypothesis is supported by the results from our study, in which we report extensive amyloid infiltration of TTR type in the atrial walls of 5 explanted hearts obtained from patients with ATTRCM. In the atrial samples, TTR amyloid deposition was associated with disruption of the normal tissue architecture, abnormalities of myocyte morphology including attenuation/atrophy, thinning and fragmentation, vascular amyloid deposits with vessel remodelling and a decrease in the capillary network. Importantly, proteomic analysis, as well as immunohistochemistry, confirmed that amyloid was of TTR type and not ANP amyloid. These results substantiate a primary contribution of interstitial and subendocardial TTR amyloid deposits to the structural changes within the atrial wall, supporting the hypothesis of primary atrial failure associated with TTR amyloid deposition rather than a phenomenon which is predominantly secondary to LV systolic and diastolic dysfunction. This hypothesis is also supported by the results of the linear regression for LA reservoir function (Supplemental Table 2), which showed a very low ability of LV diastolic variables in the prediction of the LA mechanics.

For decades, few studies have focused on the role of atrial chambers in the pathophysiology of cardiac amyloidosis, with the vast majority of studies focusing on the degree and consequences of amyloid infiltration within the ventricles.(22) Our findings suggest that atrial amyloid infiltration with associated increase in LA stiffness is an important component of overall cardiac performance, 
being independently associated with prognosis after adjusting for all known independent predictors, including all the deformation and non-deformation based ventricular structural and functional parameters. Of note atrial stiffness was an independent predictor of prognosis, whilst atrial dilatation was not in the multivariable analysis. This finding mirrors the accepted pathophysiological model associated with ventricular amyloid infiltration, in which progressive extracellular amyloid infiltration increases myocardial stiffness and results in concentric remodelling with small non-compliant chambers. Remarkably, this specific LA remodelling differs from the typical changes observed in $\mathrm{HF}$ with reduced $\mathrm{EF}$ or severe MR, where the LA dilatation is the predominant characteristic followed by reservoir disfunction and stiffness increase. In ATTR$\mathrm{CM}$ the increased stiffness and the consequent loss of distensibility prevent atrial dilatation, making the atrial dysfunction a better marker of atrial infiltration compared to atrial dimensions.

Increased LA stiffness is associated with a deterioration in the three atrial phasic function components, i.e. progressive decline in the LA reservoir, conduit and contraction phase (Figure 3). During ventricular systole, the atrial chamber acts as a non-distensible reservoir, increasing LA pressures and reducing the energy stored in the walls. The latter affects the conduit phase leading to a lower energy restitution to the blood flow during the ventricular diastole. LA infiltration affects the active phase in late ventricular diastole when the atrial contraction can be reduced or, in more severe cases, absent. Remarkably, about one fifth of patients in SR showed no evidence of atrial mechanical contraction on strain analysis, despite the presence of $\mathrm{p}$ wave on the 12-lead ECG, a form of AEMD. The concept of AEMD has already been reported in cardiac amyloidosis (23)(24), however this is the first systematic assessment in a large population of patients with ATTR amyloidosis. Patients in SR with and without atrial mechanical contraction showed distinct phenotypes, with patients in SR and no mechanical contraction having a significantly worse clinical and echocardiographic phenotype compared to patients in SR whose atrial mechanical contraction was preserved. But the importance of AEMD goes beyond echocardiographic curiosity. Patients in SR with no atrial contraction have a significantly worse prognosis compared to patients in SR with 
mechanical contraction, likely reflecting differences in the haemodynamic contribution of LA contraction to the overall cardiac performance, to a degree comparable with patients in AF. Finally, as is the case in AF, impaired atrial contraction in SR is associated with increased risk of thromboembolisation,(25)'(26) and prophylactic anticoagulation may therefore be warranted in patients with ATTR amyloidosis in whom atrial mechanical failure is documented by atrial strain analysis.

\section{Limitations}

Atrial strain was analysed retrospectively, with $26.9 \%$ of cases resulting unsuitable for the analysis. However, this is the largest cohort explored so far using this technique, providing important insight on the clinical relevance of atrial strain measurement in cardiac ATTR amyloidosis. Strain analysis was performed using a single vendor software (Echo PAC software, GE), and we acknowledge that inter-vendor variability has been reported. We do not present reproducibility in this cohort, however intra- and inter-observer reproducibility for atria strain analysis has already been published.(27) We do not have echo data on the 5 explanted hearts. Data on development of AF or rate of AF ablation procedures, although the latter is likely to be very low, are not available in this population. Further studies focusing on the relationship between atrial dysfunction and occurrence of AF will be needed to explore this point. Finally, the incidence of thromboembolic events during the follow up is not known in this population. This is a very interesting field that will need further studies.

\section{CONCLUSIONS}

In cardiac ATTR amyloidosis, extensive amyloid deposits accumulate not only in the ventricles but also within the LA, associated with loss of normal tissue architecture, and vessel remodelling. LA stiffness emerged in this study as a new independent prognostic marker, associated with reduction of reservoir, conduit and contraction functions. The use of myocardial deformation analysis allowed the identification of AEMD, a distinct clinical phenotype associated with a poor prognosis, and one meriting consideration of anticoagulation. 


\section{PERSPECTIVES}

Competency in Medical Knowledge: The LA involvement in ATTR-CM is not limited to chamber dilatation but implies the loss of physiological function (reservoir, conduit and contraction) related to increased stiffness chamber.

Competency in Patient Care: The LA strain, as assessed by echocardiography, is a reliable method to quantify the contractile function. This approach, matched with ECG rhythm analysis, helps in identifying patients with electro-mechanical dissociation (loss of contraction despite $\mathrm{P}$ wave at ECG) at increased risk of death.

Translational Outlook: The LA infiltration occurring in ATTR-CM impacts on the wall structure, physical properties (i.e. stiffness) and phasic functions of the chamber. The stages of atrial remodelling are associated with the risk of death. Further studies are needed to explore the expected link with heart failure and thromboembolic events, typically affecting ATTR-CM, and to expand the indications for thromboembolic prophylactic therapy. 


\section{REFERENCES}

1. Maceira AM, Joshi J, Prasad SK, et al. Cardiovascular magnetic resonance in cardiac amyloidosis. Circulation 2005;111:186-193.

2. Fontana M, Banypersad SM, Treibel TA, et al. Native T1 mapping in transthyretin amyloidosis. JACC Cardiovasc. Imaging 2014;7:157-165.

3. Rapezzi C, Quarta CC, Guidalotti PL, et al. Role of 99mTc-DPD scintigraphy in diagnosis and prognosis of hereditary transthyretin-related cardiac amyloidosis. JACC Cardiovasc. Imaging 2011;4:659-670.

4. Maurer MS, Bokhari S, Damy T, et al. Expert Consensus Recommendations for the Suspicion and Diagnosis of Transthyretin Cardiac Amyloidosis. Circ. Hear. Fail. 2019;12.

5. Gillmore JD, Maurer MS, Falk RH, et al. Nonbiopsy diagnosis of cardiac transthyretin amyloidosis. Circulation 2016;133:2404-2412.

6. Lane T, Fontana M, Martinez-Naharro A, et al. Natural History, Quality of Life, and Outcome in Cardiac Transthyretin Amyloidosis. Circulation 2019;140:16-26.

7. Chacko L, Martone R, Cappelli F, Fontana M. Cardiac Amyloidosis: Updates in Imaging. Curr. Cardiol. Rep. 2019;21.

8. Fontana M, Pica S, Reant P, et al. Prognostic Value of Late Gadolinium Enhancement Cardiovascular Magnetic Resonance in Cardiac Amyloidosis. Circulation 2015;132:1570-1579.

9. Martinez-Naharro A, Treibel TA, Abdel-Gadir A, et al. Magnetic Resonance in Transthyretin Cardiac Amyloidosis. J. Am. Coll. Cardiol. 2017;70:466-477.

10. Chacko L, Martone R, Bandera F, et al. Echocardiographic phenotype and prognosis in transthyretin cardiac amyloidosis. Eur. Heart J. 2020;41:1439-1447.

11. Knight DS, Zumbo G, Barcella W, et al. Cardiac Structural and Functional Consequences of Amyloid Deposition by Cardiac Magnetic Resonance and Echocardiography and Their Prognostic Roles. JACC Cardiovasc. Imaging 2019;12:823-833.

12. Bisbal F, Baranchuk A, Braunwald E, Bayés de Luna A, Bayés-Genís A. Atrial Failure as a 
Clinical Entity: JACC Review Topic of the Week. J. Am. Coll. Cardiol. 2020;75:222-232.

13. Thomas L, Marwick TH, Popescu BA, Donal E, Badano LP. Left Atrial Structure and Function, and Left Ventricular Diastolic Dysfunction: JACC State-of-the-Art Review. J. Am. Coll. Cardiol. 2019;73:1961-1977.

14. Kurt M, Wang J, Torre-Amione G, Nagueh SF. Left atrial function in diastolic heart failure. Circ. Cardiovasc. Imaging 2009;2:10-15.

15. Aquaro GD, Morini S, Grigoratos C, et al. Electromechanical dissociation of left atrium in patients with Cardiac Amyloidosis by Magnetic Resonance: Prognostic and clinical correlates. IJC Hear. Vasc. 2020;31.

16. Nochioka K, Quarta CC, Claggett B, et al. Left atrial structure and function in cardiac amyloidosis. Eur. Heart J. Cardiovasc. Imaging 2017;18:1128-1137.

17. Mohty D, Boulogne C, Magne J, et al. Prognostic value of left atrial function in systemic lightchain amyloidosis: A cardiac magnetic resonance study. Eur. Heart J. Cardiovasc. Imaging 2016;17:961-969.

18. Badano LP, Kolias TJ, Muraru D, et al. Standardization of left atrial, right ventricular, and right atrial deformation imaging using two-dimensional speckle tracking echocardiography: A consensus document of the EACVI/ASE/Industry Task Force to standardize deformation imaging. Eur. Heart J. Cardiovasc. Imaging 2018;19:591-600.

19. Sugimoto T, Robinet S, Dulgheru R, et al. Echocardiographic reference ranges for normal left atrial function parameters: Results from the EACVI NORRE study. Eur. Heart J. Cardiovasc. Imaging 2018;19:630-638.

20. Klein AL, Hatle LK, Taliercio CP, et al. Prognostic significance of Doppler measures of diastolic function in cardiac amyloidosis. A Doppler echocardiography study. Circulation 1991;83:808-816.

21. Plehn JF, Southworth J, Cornwell GGI. Atrial systolic failure in primary amyloidosis. 1992;327:1570-1572. 
22. Martinez-Naharro A, Baksi AJ, Hawkins PN, Fontana M. Diagnostic imaging of cardiac amyloidosis. Nat. Rev. Cardiol. 2020:1-14.

23. Dubrey S, Pollak A, Skinner M, Falk RH. Atrial thrombi occurring during sinus rhythm in cardiac amyloidosis: Evidence for atrial electromechanical dissociation. Heart 1995;74:541-544. 24. Stables RH, Ormerod OJ. Atrial thrombi occurring during sinus rhythm in cardiac amyloidosis: evidence for atrial electromechanical dissociation. Heart 1996;75:426.

25. Leung M, van Rosendael PJ, Abou R, et al. Left atrial function to identify patients with atrial fibrillation at high risk of stroke: new insights from a large registry. Eur. Heart J. 2017;39:14161425.

26. Martinez-Naharro A, Gonzalez-Lopez E, Corovic A, et al. High Prevalence of Intracardiac Thrombi in Cardiac Amyloidosis. J. Am. Coll. Cardiol. 2019;73:1733-1734.

27. Oxborough D, George K, Birch KM. Intraobserver reliability of two-dimensional ultrasound derived strain imaging in the assessment of the left ventricle, right ventricle, and left atrium of healthy human hearts. Echocardiography 2012;29:793-802. 


\section{FIGURES TITLES AND CAPTIONS}

Figure 1. Histological findings in post-mortem atria.

Atrial sample histology from native hearts of a 71-year-old male with V122I associated hATTR$\mathrm{CM}(\mathrm{A}, \mathrm{E}, \mathrm{F})$, of a 85-year-old-male with wtATTR-CM(B,C,D,H), of a 51-year-old male transplanted for wtATTR-CM $(\mathrm{G})$ and of a 54-year-old male with E89Q associated hATTR-CM(I). A-D: Scanner magnification shows the extensive eosinophilic amyloid deposits distributed throughout subendocardium and myocardial interstitium, in the form of both streaks along myocytes and large accumulations (A:H\&E 25x;B:H\&E 25x;C:Congo red green birefringence 25x;D:TTR immunohistochemistry,25x).E and $\mathrm{F}(\mathrm{H} \& \mathrm{E}, 100 \mathrm{x})$ highlight nodular amyloid deposits responsible for attenuation/atrophy (black asterisks) or thinning out (red asterisks) of myocytes. G:At high power (H\&E,200x) cytoplasmic vacuolization of myocytes are evident. H(Congo red,100x):Green amyloid deposits are present within the wall of a small intramural artery (white arrow). I:CD31 immunostaining shows decreased/absent capillary network within nodular amyloid aggregates (asterisks) (100x).

Figure 2. LA reservoir, conduit and contraction function in patients with ATTR-CM according to increasing LA stiffness.

Median with interquartile range are represented; $p$ value refers to adjusted pairwise comparisons.

Figure 3. LA mechanics in in patients with sinus rhythm with left atrial mechanical contraction (SR with LAMC), sinus rhythm without left atrial mechanical contraction (SR without LAMC) and non-sinus rhythm (Non-SR).

From top to bottom: apical 4 chamber view showing strain endocardial trace; endocardial longitudinal strain curves with measurements of LA functional components; transmitral PW Doppler; ECG trace.

Figure 4. Kaplan-Meier curves displaying the prognostic impact of left atrial stiffness (LA stiffness) in patients with ATTR-CM (left panel).

Kaplan Meier curves displaying the differences in prognosis in patients with sinus rhythm with left atrial mechanical contraction (SR with LAMC), sinus rhythm without left atrial mechanical contraction (SR without LAMC) and non-sinus rhythm (Non-SR).

Central figure. Clinical importance of LA infiltration in ATTR-CM.

Main histological, echocardiographic, mechanical, Doppler, ECG and survival characteristics according with the 3 rhythm phenotypes. 


\section{TABLES}

Table 1: Clinical characteristics in the 3 subgroups: SR with LAMC, SR without LAMC and Non-SR.

\begin{tabular}{|c|c|c|c|c|}
\hline Clinical variables & $\begin{array}{l}\text { SR } \\
\text { with LAMC } \\
n=439(48.5 \%)\end{array}$ & $\begin{array}{l}\text { SR without } \\
\text { LAMC } \\
n=125(13.8 \%)\end{array}$ & $\begin{array}{l}\text { Non-SR } \\
n=342(37.7 \%)\end{array}$ & $\mathrm{p}$ value \\
\hline Age, years & $74(8.5)$ & $75.3(8.2)$ & $76.9(6.8)$ & $<0.001$ \& \\
\hline Sex(male) & $353(80.4 \%)$ & $104(83.2 \%)$ & $315(92.2 \%)$ & $<0.001$ \\
\hline BSA, $\mathrm{kg} / \mathrm{m}^{2}$ & $1.86(0.19)$ & $1.86(0.19)$ & $1.90(0.17)$ & $0.007 \S$ \\
\hline Race & & & & $<0.001$ \\
\hline Black & $128(29.1 \%) \S$ & $41(32.8 \%)^{\circ}$ & $67(19.7 \%)$ & \\
\hline Caucasian & $283(64.5 \%) \S$ & $76(60.8 \%)^{\circ}$ & $265(77.5 \%)$ & \\
\hline Other & $19(4.4 \%) \S$ & $5(4 \%)^{\circ}$ & $2(0.5 \%)$ & \\
\hline Missing & $9(2.0 \%)$ & $3(2.4 \%)$ & $8(2.3 \%)$ & \\
\hline Genotype & & & & $<0.001$ \\
\hline Wildtype & $236(53.7 \%) \S$ & $61(48.8 \%)^{\circ}$ & $254(74.3 \%)$ & \\
\hline T60A & $59(13.4 \%) \S$ & $13(10.4 \%)$ & $21(6.1 \%)$ & \\
\hline V122I & $129(29.4 \%) \S$ & $48(38.4 \%)^{\circ}$ & $64(18.7 \%)$ & \\
\hline Other variants & $15(3.5 \%)$ & $3(2.4 \%)$ & $3(0.9 \%)$ & \\
\hline NYHA class & & & & 0.005 \\
\hline 1 & $51(11.6 \%) \S$ & $7(5.6 \%)$ & $17(5.0 \%)$ & \\
\hline 2 & $313(71.3 \%)$ & $92(73.6 \%)$ & $241(70.5 \%)$ & \\
\hline 3 & $74(16.9 \%)$ & $25(20.0 \%)$ & $80(23.4 \%)$ & \\
\hline 4 & $1(0.2 \%)$ & $1(0.8 \%)$ & $4(1.2 \%)$ & \\
\hline Biomarker stage & & & & $<0.001$ \\
\hline
\end{tabular}




\begin{tabular}{|c|c|c|c|c|}
\hline Grade 1 & $259(59.0 \%) * \S$ & $54(43.9 \%)$ & $113(32.8 \%)$ & \\
\hline Grade 2 & $124(28.2 \%) \S$ & $40(32.5 \%)^{\circ}$ & $164(47.7 \%)$ & \\
\hline Grade 3 & $51(11.6 \%) * \S$ & $29(23.6 \%)$ & $64(18.6 \%)$ & \\
\hline Missing data & $5(1.2 \%)$ & $0(0 \%)$ & $3(0.9 \%)$ & \\
\hline Heart Rate, bpm & $70(12)$ & $75(15)$ & $75(15)$ & $<0.001 * \S$ \\
\hline Systolic Blood Pressure, $\mathrm{mmHg}$ & 127(19) & $125(18)$ & $121(17)$ & $<0.001$ \& \\
\hline Diastolic Blood Pressure, $\mathrm{mmHg}$ & $74(10)$ & $74(11)$ & $74(11)$ & 0.911 \\
\hline NTproBNP, ng/L & $2064(1108-3755)$ & $3696(1937-6484)$ & $3856(2287-6614)$ & $<0.001 * \S$ \\
\hline Troponin, ng/mL & $53(35-81)$ & $71(48-104)$ & $64(42-95)$ & $<0.001 * \xi^{\circ}$ \\
\hline $\mathrm{GFR}, \mathrm{mL} / \mathrm{min} / 1.73 \mathrm{~m}^{2}$ & $63(50-79)$ & $56(44-67)$ & $56(46-70)$ & $<0.001 * \S$ \\
\hline 6MWT, meters & $348(138)$ & $310(140)$ & $309(136)$ & $0.010 * \S$ \\
\hline 6MWT, \%of predicted & $77(27)$ & $71(27)$ & $66(26)$ & $0.009 \S$ \\
\hline Antiplatelet treatment & $83(19)$ & $19(15)$ & $24(7)$ & $<0.001^{\circ} \S$ \\
\hline Anticoagulants & $114(26)$ & $34(27)$ & $196(57)$ & $<0.001^{\circ} \S$ \\
\hline Warfarin & $65(15)$ & $16(13)$ & $137(40)$ & $<0.001^{\circ} \S$ \\
\hline DOAC & $49(11)$ & $18(14)$ & $59(17)$ & 0.225 \\
\hline ACEIs & $154(44)$ & $49(46)$ & $143(42)$ & 0.911 \\
\hline ARBs & $63(18)$ & $18(17)$ & $52(17)$ & 0.877 \\
\hline Beta-blockers & $167(48)$ & $52(49)$ & $179(58)$ & $0.040 \S$ \\
\hline MRA & $82(24)$ & $24(22)$ & $93(30)$ & 0.124 \\
\hline Loop diuretics & $226(65)$ & $79(74)$ & $253(81)$ & $<0.001$ \& \\
\hline Thiazide diuretics & $15(4)$ & $8(7)$ & $22(7)$ & 0.245 \\
\hline
\end{tabular}

Data are presented as means(SD), median( $25^{\text {th }}-75^{\text {th }}$ percentiles) or number(percentage). 
$\mathrm{P}$-values refer to comparison above the 3 subgroups. $\mathrm{P}$ values for pairwise comparison *: $\mathrm{p}<0.05$ for SR with LAMC vs SR without LAMC; ${ }^{\circ}: \mathrm{p}<0.05$ for SR without LAMC vs. Non-SR; $\$: \mathrm{p}<0.05$ for SR with LAMC vs Non-SR.

BSA is body surface area; NYHA New York heart association; NTproBNP N-terminal prohormone Brain Natriuretic Peptide; GFR glomerular filtration rate; 6MWT six minutes walking test; DOAC direct oral anticoagulants; ACEI Angiotensin-Converting Enzyme Inhibitor; ARB Angiotensin Receptor Blocker; MRA Mineralcorticoid Receptor Antagonist. 
Table 2: Echocardiographic findings in the 3 subgroups: SR with LAMC, SR without LAMC and Non-SR.

\begin{tabular}{|c|c|c|c|c|}
\hline Echocardiographic variables & $\begin{array}{l}\text { SR } \\
\text { with LAMC } \\
n=439(48.5 \%)\end{array}$ & $\begin{array}{l}\text { SR without } \\
\text { LAMC } \\
\mathrm{n}=125(13.8 \%)\end{array}$ & $\begin{array}{l}\text { Non-SR } \\
n=342(37.7 \%)\end{array}$ & $\mathrm{p}$ value \\
\hline $\operatorname{IVSD}(\mathrm{mm})$ & $16.7(2.4)$ & $16.8(2.2)$ & $16.9(2.3)$ & 0.262 \\
\hline PWTD $(\mathrm{mm})$ & $16.4(2.4)$ & $16.6(2.2)$ & $16.3(2.5)$ & 0.226 \\
\hline $\operatorname{LVM}(g)$ & $307.0(79.8)$ & $297.0(79.1)$ & $318.2(74.2)$ & $<0.001 \S^{\circ}$ \\
\hline RWT & $0.76(0.17)$ & $0.79(0.15)$ & $0.76(0.17)$ & 0.016* \\
\hline LVEDD(mm) & $43.4(5.8)$ & $41.7(5.5)$ & $44.0(5.8)$ & $<0.001 * 0$ \\
\hline LVEDD indexed $\left(\mathrm{mm} / \mathrm{m}^{2}\right)$ & $23.4(3.1)$ & $22.5(2.9)$ & $23.1(2.9)$ & 0.006* \\
\hline MWT(mm) & $16.2(2.3)$ & $16.7(2.1)$ & $16.6(2.3)$ & 0.242 \\
\hline $\mathrm{LVEDV}(\mathrm{ml})$ & $77.0(25.9)$ & $69.3(22.2)$ & $77.7(26.3)$ & $0.008 * \circ$ \\
\hline LVEDV indexed $\left(\mathrm{ml} / \mathrm{m}^{2}\right)$ & $41.3(12.7)$ & $37.4(10.6)$ & $40.6(12.7)$ & $0.017 *$ \\
\hline $\operatorname{LVESV}(\mathrm{ml})$ & $38.5(17.7)$ & $38.6(17.2)$ & $42.8(18.5)$ & $<0.001$ \& \\
\hline LVESV indexed $\left(\mathrm{ml} / \mathrm{m}^{2}\right)$ & $20.6(8.9)$ & $20.8(8.4)$ & $22.4(9.4)$ & $0.013 \S$ \\
\hline $\mathrm{SV}(\mathrm{ml})$ & $38.6(13.7)$ & $30.7(11.0)$ & $34.9(13.4)$ & $<0.001 * \S^{\circ}$ \\
\hline $\mathrm{SV}$ indexed $\left(\mathrm{ml} / \mathrm{m}^{2}\right)$ & $20.8(6.8)$ & $16.6(5.6)$ & $18.2(6.6)$ & $<0.001 * \S$ \\
\hline $\operatorname{LVEF}(\%)$ & $50.6(10.4)$ & $45.0(10.8)$ & $45.5(11.1)$ & $<0.001 * \S$ \\
\hline $\mathrm{E}(\mathrm{cm} / \mathrm{s})$ & $83.8(20.7)$ & $86.8(19.4)$ & $84.8(18.7)$ & 0.188 \\
\hline $\mathrm{DTE}(\mathrm{ms})$ & $187.2(55.7)$ & $163.6(57.3)$ & $177.7(52.4)$ & $<0.001 * \S$ \\
\hline $\mathrm{E} / \mathrm{A}$ & $2.11(1.6)$ & -- & -- & -- \\
\hline Mean e'(cm/s) & $5.1(1.5)$ & $5.3(1.7)$ & $5.9(1.7)$ & $<0.001 \S^{\circ}$ \\
\hline $\mathrm{E} / \mathrm{e}^{\prime}$ & $17.4(6.0)$ & $17.8(6.1)$ & $15.6(6.0)$ & $<0.001^{\circ}$ \\
\hline $\mathrm{RA}$ area $\left(\mathrm{cm}^{2}\right)$ & $22.0(5.7)$ & $24.2(5.8)$ & $27.1(6.6)$ & $<0.001 * \xi^{\circ}$ \\
\hline
\end{tabular}




\begin{tabular}{|c|c|c|c|c|}
\hline RA area indexed $\left(\mathrm{cm}^{2} / \mathrm{m}^{2}\right)$ & $12.0(3.0)$ & $13.1(2.9)$ & $14.2(3.4)$ & $<0.001 * \S^{\circ}$ \\
\hline MAPSE(mm) & $9.0(6.7)$ & $7.6(2.6)$ & $7.6(2.5)$ & $<0.001 * \S$ \\
\hline TAPSE(mm) & $16.7(4.5)$ & $13.6(4.4)$ & $12.8(3.7)$ & $<0.001 * \S$ \\
\hline PASP(mmHg) & $42.7(12.3)$ & $45.2(9.4)$ & 43.3(9.6) & 0.056 \\
\hline TAPSE/PASP $(\mathrm{mm} / \mathrm{mmHg})$ & $0.43(0.22)$ & $0.30(0.12)$ & $0.31(0.14)$ & $<0.001 * \S$ \\
\hline Tricuspid S'(cm/s) & $11.3(3.1)$ & $9.0(2.6)$ & $9.1(2.5)$ & $<0.001 * \S$ \\
\hline $\mathrm{MCF}(\%)$ & $15.9(5.9)$ & $13.9(5.0)$ & $14.3(5.2)$ & $<0.001 * \S$ \\
\hline $\operatorname{LVLS}(\%)$ & $-12.0(3.7)$ & $-9.4(3.5)$ & $-10.1(3.3)$ & $<0.001 * \S$ \\
\hline SABr & $5.35(6.63)$ & $4.78(6.53)$ & $5.20(7.16)$ & 0.611 \\
\hline RALS & $1.70(1.15)$ & $1.96(1.61)$ & $1.82(1.30)$ & 0.310 \\
\hline Significant MR & $118(26.9 \%)$ & $43(34.4 \%)$ & $133(38.9 \%)$ & $0.002 \S$ \\
\hline Significant TR & $107(24.4 \%)$ & $52(41.6 \%)$ & $128(37.2 \%)$ & $<0.001 * \S$ \\
\hline LA Diameter $(\mathrm{mm})$ & $43.7(5.5)$ & $44.2(6.0)$ & $46.6(5.4)$ & $<0.001 \S^{\circ}$ \\
\hline LA Area $\left(\mathrm{cm}^{2}\right)$ & $25.2(4.9)$ & $26.0(5.2)$ & 28.1(5.9) & $<0.001 \S^{\circ}$ \\
\hline LA Area indexed $\left(\mathrm{cm}^{2 /} \mathrm{m}^{2}\right)$ & $13.7(2.8)$ & $14.0(2.7)$ & $14.8(3.0)$ & $<0.001$ § \\
\hline LAS reservoir(\%) & $11.2(8.1-15.3)$ & $7.3(5.2-10.9)$ & $6.9(4.7-9.6)$ & $<0.001 * \S$ \\
\hline LAS conduit(\%) & $6.5(4.5-9.3)$ & -- & -- & \\
\hline LAS contraction(\%) & $4.1(2.3-6.6)$ & -- & -- & \\
\hline LA Stiffness(1/\%) & $1.47(0.98-2.39)$ & $2.30(1.40-3.54)$ & $2.16(1.38-3.54)$ & $<0.001 * \S$ \\
\hline lnLA Stiffness & $0.38(0.02-0.87)$ & $0.83(0.34-1.26)$ & $0.77(0.32-1.26)$ & $<0.001 * \S$ \\
\hline
\end{tabular}

Data are presented as means(SD), median $\left(25^{\text {th }}-75^{\text {th }}\right.$ percentiles $)$, or number(percentage).

P-values refer to comparison above the 3 subgroups. P values for pairwise comparison *:p<0.05 for SR with LAMC vs SR without LAMC; ${ }^{\circ}$ :p<0.05 for SR without LAMC vs. Non-SR; $§: p<0.05$ for SR with LAMC vs Non-SR. 
IVSD: diastolic interventricular septum; PWTD: diastolic posterior wall thickness; LVM: left ventricle mass; RWT: relative wall thickness; LVEDD: left ventricle end-diastolic diameter; MWT: maximal wall thickness; LVEDV: left ventricle end diastolic volume; LVESV: left ventricle end systolic volume; SV: stroke volume; LVEF: left ventricle ejection fraction; DTE: E deceleration time; RA: right atrium; MAPSE: mitral annular plane systolic excursion; TAPSE: tricuspid annular plane systolic excursion; PASP: pulmonary artery systolic pressure; MCF: myocardial contraction fraction; LS: longitudinal strain; SABr: systolic apex to base ratio; RALS: relative apical longitudinal strain; MR: mitral regurgitation; TR: tricuspid regurgitation; LA: left atrium; LAS: peak left atrium strain. 
Table 3. LA mechanics and stiffness in ATTR-CM genotypes.

\begin{tabular}{|l|l|l|l|l|}
\hline \multirow{2}{*}{ Echocardiographic variables } & WtATTR-CM & T60A-ATTR-CM & V122A-ATTR- & \\
& $\mathrm{n}=554(62 \%)$ & $\mathrm{n}=97(11 \%)$ & $\mathrm{n}=242(27 \%)$ & $\mathrm{p}$ value \\
\hline LAS reservoir (\%) & $9.0(6.0-13.0)$ & $11.4(7.0-15.7)$ & $8.0(5.3-10.9)$ & $\mathbf{0 . 0 0 1}^{* \circ} \S$ \\
\hline LAS conduit (\%) & $6.6(4.5-9.3)$ & $7.0(4.7-10.0)$ & $6.1(4.2-8.2)$ & 0.078 \\
\hline LAS contraction $(\%)$ & $4.1(2.3-7.0)$ & $5.0(2.8-8.4)$ & $3.1(1.6-5.0)$ & $\mathbf{0 . 0 0 1}^{\circ} \S$ \\
\hline LA Stiffness $(1 / \%)$ & $1.72(1.10-2.83)$ & $1.67(0.87-2.70)$ & $2.12(1.34-3.29)$ & $\mathbf{0 . 0 0 1}^{\circ} \S$ \\
\hline
\end{tabular}

Data are presented as median $\left(25^{\text {th }}-75^{\text {th }}\right.$ percentiles $)$.

$\mathrm{P}$-values refer to comparison above the 3 subgroups. $\mathrm{P}$ values for pairwise comparison $*$ : $\mathrm{p}<0.05$ for

WtATTR-CM vs T60A-ATTR-CM; ${ }^{\circ}$ p $<0.05$ for T60A-ATTR-CM vs. V122A-ATTR-CM; $§: p<0.05$ for WtATTR-CM vs V122A-ATTR-CM 
Table 4. Multivariable Cox Regression Analysis of Risk of Death.

\begin{tabular}{|c|c|c|}
\hline Variables in the model & Multivariable & \\
\hline & $\mathrm{HR}(95 \% \mathrm{CI})$ & $\mathrm{p}$ value \\
\hline IVSD & $1.00(0.93-1.09)$ & 0.864 \\
\hline RWT & $0.80(0.29-2.19)$ & 0.665 \\
\hline SVindexed & $0.98(0.96-1.00)$ & 0.120 \\
\hline LVLS (\%) & $1.07(1.03-1.12)$ & 0.002 \\
\hline Significant TR & $0.98(0.73-1.31)$ & 0.90 \\
\hline Significant MR & $1.35(1.03-1.77)$ & 0.032 \\
\hline RA Area indexed & $1.05(1.01-1.10)$ & 0.033 \\
\hline TAPSE/PASP & $1.73(0.75-3.96)$ & 0.195 \\
\hline T60A vs Wildtype & $1.25(0.81-1.94)$ & 0.314 \\
\hline V122I vs Wildtype & $1.49(1.12-1.97)$ & 0.006 \\
\hline LA Area indexed & $1.01(0.96-1.06)$ & 0.725 \\
\hline lnLA stiffness & $1.23(1.03-1.49)$ & 0.029 \\
\hline Heart Rate & $0.99(0.99-1.01)$ & 0.827 \\
\hline Non-SR vs SR & $1.14(0.88-1.47)$ & 0.312 \\
\hline $\begin{array}{l}\text { Diagnosis before vs after } 09 \text { February } \\
2015\end{array}$ & $1.52(1.13-2.06)$ & 0.006 \\
\hline
\end{tabular}

Data is presented as hazard ratio (HR) and 95\% confidence interval. 
SUPPLEMENTAL MATERIAL

Supplemental Table 1. Univariate Cox Regression Analysis of Risk of Death

\begin{tabular}{|c|c|c|}
\hline Variables in the model & \multicolumn{2}{|l|}{ Univariate } \\
\hline & $\mathrm{HR}(95 \% \mathrm{CI})$ & $\mathrm{p}$ value \\
\hline IVSD & $1.07(1.03-1.12)$ & 0.002 \\
\hline RWT & $2.19(1.19-4.03)$ & 0.011 \\
\hline SVindexed & $0.95(0.94-0.97)$ & $<0.001$ \\
\hline LVEF & $0.97(0.96-0.98)$ & $<0.001$ \\
\hline LVLS (\%) & $1.11(1.07-1.14)$ & $<0.001$ \\
\hline $\mathrm{E} / \mathrm{e}^{\prime}$ & $1.02(1.01-1.05)$ & 0.003 \\
\hline $\mathrm{MCF}$ & $0.95(0.93-0.97)$ & $<0.001$ \\
\hline MAPSE & $0.93(0.90-0.97)$ & $<0.001$ \\
\hline Significant TR & $1.56(1.27-1.92)$ & $<0.001$ \\
\hline Significant MR & $1.50(1.22-1.85)$ & $<0.001$ \\
\hline RA Area indexed & $1.09(1.06-1.12)$ & $<0.001$ \\
\hline TAPSE & $0.94(0.92-0.96)$ & $<0.001$ \\
\hline TAPSE/PASP & $0.36(0.18-0.70)$ & 0.002 \\
\hline T60A vs Wildtype & $1.10(0.78-1.55)$ & 0.575 \\
\hline V122I vs Wildtype & $1.71(1.36-2.15)$ & $<0.001$ \\
\hline LA Diameter & $1.01(0.99-1.03)$ & 0.367 \\
\hline LA Area & $1.02(1.00-1.04)$ & 0.047 \\
\hline LA Area indexed & $1.07(1.03-1.10)$ & $<0.001$ \\
\hline LAS reservoir & $0.95(0.93-0.97)$ & $<0.001$ \\
\hline
\end{tabular}




\begin{tabular}{|l|l|l|}
\hline LAS contraction & $0.93(0.89-0.97)$ & $\mathbf{0 . 0 0 3}$ \\
\hline LAS conduit & $0.99(0.95-1.03)$ & 0.545 \\
\hline InLA stiffness & $1.51(1.32-1.73)$ & $<\mathbf{0 . 0 0 1}$ \\
\hline Heart Rate & $1.00(1.00-1.02)$ & 0.073 \\
\hline Non-SR vs SR & $1.26(1.02-1.55)$ & $\mathbf{0 . 0 2 9}$ \\
\hline Diagnosis before vs diagnosis after 09 February 2015 & $1.88(1.44-2.44)$ & $<\mathbf{0 . 0 0 1}$ \\
\hline
\end{tabular}

Data are presented as hazard ratio (HR) and 95\% confidence interval. 
Supplemental Table 2. Linear regression for determinants of LA reservoir function.

\begin{tabular}{lllllll} 
Variable & $\mathbf{B}$ & $\mathbf{9 5 \%} \mathbf{C I}$ & $\boldsymbol{\beta}$ & $\mathbf{t}$ & $\mathbf{p}$ & $\mathbf{R}$ adj \\
\hline E wave & & & & & & \\
A wave & .030 & {$[-.050,-.009]$} & -.093 & -2.798 & .005 & .089 \\
DTE & .109 & {$[.088, .130]$} & .397 & 10.242 & $<.001$ & .394 \\
E/A & -1.025 & {$[.018, .033]$} & .222 & 6.787 & $<.001$ & .219 \\
Mean e' & .747 & {$[-1.374,-.662]$} & -.230 & -5.616 & $<.001$ & .225 \\
E/mean e' & -.227 & {$[-.293,-.161]$} & -.222 & -6.723 & $<.001$ & .219 \\
LA indexed area & -.669 & {$[-.803,-.535]$} & -.315 & -9.79 & $<.001$ & .313 \\
LV LS & -.805 & {$[-.906,-.704]$} & -.469 & -15.656 & $<.001$ & .467 \\
LV indexed mass & -.204 & {$[-.035,-.014]$} & -.149 & -4.445 & $<.001$ & .145 \\
Age & -.143 & {$[-.194,-.093]$} & -.182 & -5.566 & $<.001$ & .179
\end{tabular}

Multivariable

Model

Age $\quad-.106 \quad[-.172,-.040] \quad-.129 \quad-3.151 \quad .002$

E/A $\quad-.803 \quad[-1.144,-.461] \quad-.183 \quad-4.618 \quad<.001$

LA indexed area $\quad-.399 \quad[-.603,-.195] \quad-.163 \quad-3.837 \quad<.001$

E/e' $\quad-.223 \quad[-.317,-.129] \quad-.197 \quad-4.659 \quad<.001$

LV ind mass $\quad-.014 \quad[-.028,-.001] \quad-.078 \quad-1.872 \quad .062$

Abbreviations as in the text. 